\title{
RESEARCH
}

\section{Generation of a High-resolution Genetic Map and a YAC Contig of the Lurcher Locus on Mouse Chromosome 6}

\author{
Jian Zuo, Philip L. De Jager, Deborah J. Norman, ${ }^{1}$ \\ and Nathaniel Heintz ${ }^{2}$
}

Howard Hughes Medical Institute, The Rockefeller University, New York, New York 10021

\begin{abstract}
Lurcher $(L C)$ is a semidominant mouse mutant that displays progressive neurodegeneration during perinatal development. This genetic lesion results in apoptotic neuronal death in a dosage dependent and cell autonomous manner in specific neurons during their terminal differentiation. To understand the molecular basis of the Lc mutation, we have adopted a positional cloning approach based on its location on mouse chromosome 6. To define the $L c$ locus, we have extended our previous analysis of an intersubspecific backcross between Mus m. castaneus and B6CBACa- $\mathrm{A}^{\mathrm{w}-\mathrm{I}} / \mathrm{A}-\mathrm{Lc}$ consisting of 504 animals (Norman et al. 1991). In addition, 580 animals of a generic backcross between Mus spretus and C57BL/ 6 (The European Collaborative Interspecific Backcross) were utilized for the fine genetic mapping of the $L c$ locus. Using three RFLP markers and nine microsatellite markers in the vicinity of the $L c$ locus, we determined the order and relative genetic distances of these markers at a resolution of $0.1 \mathrm{cM}$. The $L c$ mutation was mapped between two flanking markers, D6Miti2I and D6Mit175, separated by a genetic distance of $0.5 \mathrm{cM}$. We then initiated the cloning of the genomic region surrounding these two markers by screening a YAC library and characterizing YAC end sequences for further screening. This effort has resulted in the construction of a YAC contig consisting of 14 YACs and spanning a $3-\mathrm{Mb}$ region. Markers isolated from these YACs were used to further define the $L c$ locus, resulting in a physical map that places the $L c$ gene within an estimated $300-\mathrm{kb}$ interval. This set of YACs and markers will serve as DNA sources for the identification of the $L c$ gene.
\end{abstract}

Lurcher $(L c)$ is a semidominant mouse neurological mutant that affects cerebellar development (Phillips 1960). Beginning at postnatal day 14 (P14), heterozygous $L c /$ +animals display locomotor difficulties that include an ataxic gait and a tendency to walk backwards. In contrast, homozygous $L c / L C$ animals die within a few hours of birth with no gross anatomic abnormalities (Phillips 1960). The phenotypic consequences of the $L c$ mutation have been studied in detail in $L c /$ +heterozygous animals. Anatomical analysis of adult $L c /+$ heterozygotes reveals a marked reduction in cerebellar volume but no other gross defect in organogenesis. Detailed histological studies of the cerebella of $L c /+$ animals have revealed massive cerebellar cell death and have established a chronology for this neurodegeneration. The Purkinje cells (PCs) degenerate during

\footnotetext{
'Present address: Howard Hughes Medical Institute, University of California at San Diego, La Jolla, California 92093. ${ }^{2}$ Corresponding author. E-MAIL HEINTZ@ROCKVAX. ROCKEFELLER.EDU; FAX (212)327-7878.
}

the second postnatal week, and their disappearance is followed by the progressive loss of $-90 \%$ of cerebellar granule cells and $75 \%$ of inferior olivary neurons and Bergmann glia (Caddy and Biscoe 1979). Studies of $L c$ and wild-type chimeric mice have defined the action of the $L c$ gene as being cell autonomous; its primary site of action lies within PCs (Wetts and Herrup 1982a,b,c; 1983). The degeneration of other cerebellar cell types is a secondary effect, presumably owing to the lack of essential contacts with PCs. The death of $L c / L c$ homozygotes within a few hours of birth led us to search for lesions in the respiratory, digestive, and nervous systems of the newborn $L c$ / $L c$ mice. Whereas these systems are mostly unaffected, the hindbrain is significantly reduced in both size and cell density at P0. The widespread neuronal cell death that causes this reduction is most clearly illustrated by the loss of large neurons in the motor nuclei of the fifth cranial nerves beginning at embryonic day 16 (E16) (S. Cheng and N. Heintz, in prep.). By combining the results of the analyses of $L c$ heterozygous and 


\section{ZUO ET AL.}

homozygous animals, we conclude that the $L c$ mutation is involved in the degeneration of specific populations of neurons as they mature in the developing central nervous system and that the dosage of the mutant gene determines which neuronal populations are affected.

Recently, the mechanisms of neuronal death in $L c$ animals have been studied extensively (Norman et al. 1995). Electron microscopy of $L c /+\mathrm{PCs}$ at $\mathrm{P} 12$ reveals chromatin condeñsation in the nucleus and membrane blebbing, two ultrastructural hallmarks of apoptotic cell death. In addition, $L c /+P C s$ but not wild-type PCs display double-stranded DNA breaks in their nuclei and expression of sulfated glycoprotein-2, a gene that is up-regulated in certain forms of apoptosis. Therefore, we have concluded that the PCs in $L C l$ tanimals die by apoptosis. Furthermore, degeneration of large neurons in the motor nuclei of the fifth cranial nerves in $L c / L c$ animals is also attributable to apoptosis ( $\mathrm{S}$. Cheng and $\mathrm{N}$. Heintz, in prep.). Therefore, the isolation of the $L c$ gene may provide insights into the molecular mechanisms involved in activation of programmed cell death.

To identify the $L c$ gene and to understand its possible roles during the development and degeneration of the mammalian nervous system, we have adopted a positional cloning strategy. This approach takes advantage of mouse genetics and molecular cloning technology to identify the mutant gene based on its chromosomal location. The $L c$ mutation arose spontaneously in 1954 in one of the coat color mutant colonies, $\mathrm{Mi}^{\mathrm{wh}}$ (Phillips 1960); $M i^{\text {wh }}$ was itself found in a cross between the DBA and C57BL strains (Grobman and Charles 1947). $L c$ was then backcrossed to and maintained on a $\mathrm{C} 3 \mathrm{HeB} / \mathrm{FeJ}$ strain background in Harwell, England (Caddy and Biscoe 1979). This mutant was then crossed to a B6CBACa-A ${ }^{\text {w- }} / \mathrm{A}$ F1 hybrid in the Jackson Laboratory in 1976 and was maintained further in such a hybrid background for $>50$ generations $(H$. Sweet, pers. comm.). Therefore, a total of four inbred strains may have contributed to the genetic background in the vicinity of the $L c$ mutation. $L c$ has been genetically mapped to mouse Chromosome 6 and has a single known allele (Phillips 1960). In a previous report, an intersubspecific backcross between Mus $m$. castaneus (CAST/Ei) and B6CBACa-A ${ }^{\mathrm{w}-\mathrm{J}} / \mathrm{A}-L c$ (Lc/cast.) was initiated and 504 progeny were analyzed by using six polymorphic DNA markers on Chromosome 6 (Norman et al. 1991). As a result, the $L c$ locus was mapped to an interval of $-2.5 \mathrm{cM}$. This segment has no known synteny to the human genome. The closest markers that have established human homologs are Ghrhr, $\sim 2 \mathrm{cM}$ centromeric to $L c$, and Igk, $\sim 2 \mathrm{cM}$ telomeric to $L c$ (Norman et al. 1991; Chua et al. 1993). Ghrhr maps to human 7p14, and Igk maps to human 2p12 (Gaylinn et al. 1994; McBride et al. 1982).

In this report we have continued our analysis of this set of $L c /$ cast. backcross progeny and of another large set of mice from a generic backcross, the European Collaborative Interspecific Backcross (EUCIB) between M. spretus and $\mathrm{C} 57 \mathrm{BL} / 6$, with markers in the vicinity of the $L c$ locus. A high resolution genetic map, a YAC contig, and a high density of novel markers in the $L c$ region are reported here. These tools will facilitate the isolation of candidate genes for $L c$.

\section{RESULTS}

\section{A High-Resolution Genetic Map of the Lc Region}

In a previously established phenotypic backcross between B6CBACa-A $\mathrm{A}^{\mathrm{w}-\mathrm{J}} / \mathrm{A}-L c$ and $M$. m. castaneus, a total of 504 animals have been genetically typed with markers on either side of the $L c$ locus. This analysis has localized the $L c$ mutation between the centromeric marker D6Rkf41 and the two telomeric markers, D6Rkf33 and D6Rkf39, which are nonrecombinant with each other (Norman et al. 1991, 1995; J. Zuo and N. Heintz, unpubl.). There are a total of six recombinants between $D 6 R k f 41$ and $D 6 R k f 33 / D 6 R k f 39$, indicating that the $L c$ region, defined by D6Rkf41 and D6Rkf33/D6Rkf39, has a genetic distance of $\sim 1.2$ cM. These recombinants are shown in Figure 1B, which also includes a number of additional markers described later.

To resolve the exact locations of these six crossover events, we utilized the collection of polymorphic markers developed at the Massachusetts Institute of Technology (MIT) (Dietrich et al. 1994). Using eight MIT markers known to map to the appropriate region of mouse Chromosome 6, we first typed 21 animals with recombination events near $L c$, including the six critical recombinants between D6Rkf41 and D6Rkf33/ D6Rkf39 (Fig. 1; data not shown). All eight MIT markers are closely linked to $L c$ in these 21 recombinant animals and can be mapped either centromeric or telomeric to $L c$. Three of the six 
A YAC CONIIG OF IHE LURCHER LOCUS

A
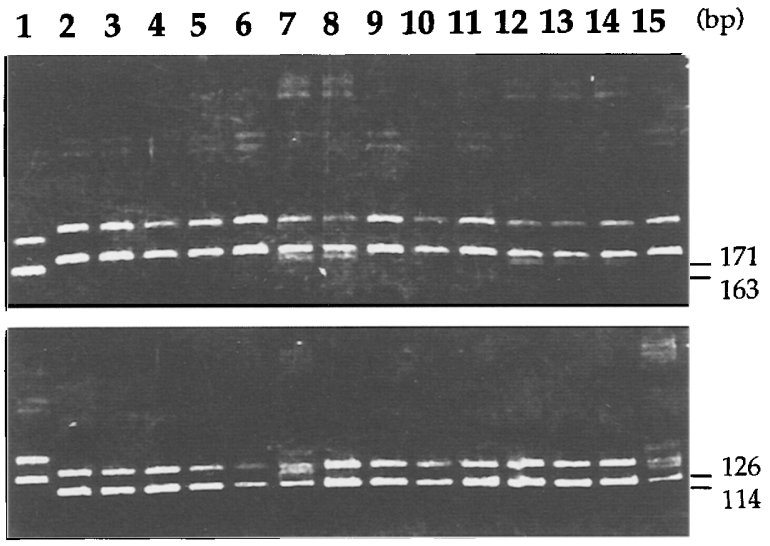

B

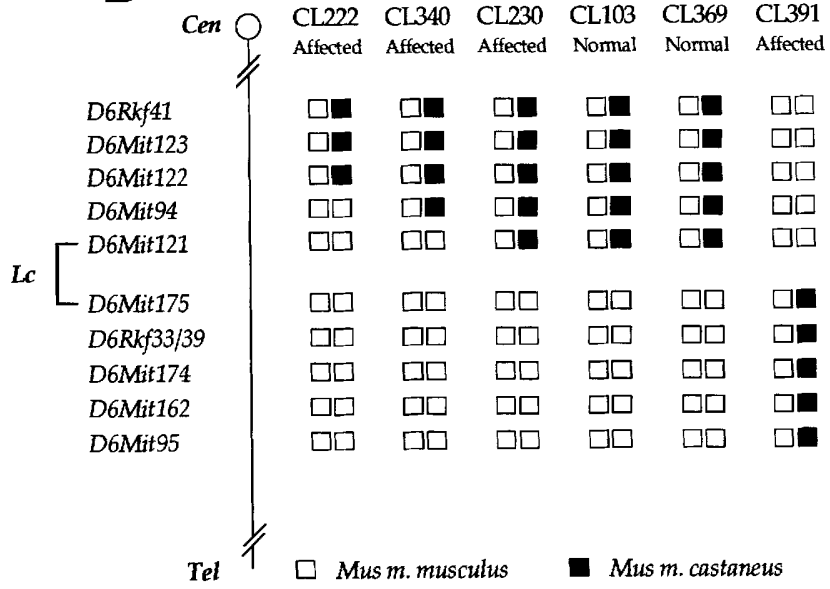

Figure $1 P C R$ analyses with two MIT markers on DNA samples from the $L C /$ cast. backcross and genotypes of critical recombinant animals in the $L C$ region on Chromosome 6 . The $L C / c a s t$. backcross was generated by crossing $B 6 C B A C a-A^{w-1} / A-L c$ with $M$. m. castaneus, selecting $F_{1}$ progeny displaying the $L c$ phenotype and backcrossing them to B6CBACa- $A^{\mathrm{w}-1} / \mathrm{A}$ mice. (A) Products derived from PCR amplification with two of the MIT markers used, D6Mit94 (top) and D6Mit95 (bottom), following polyacrylamide gel electrophoresis and staining with ethidium bromide. DNA samples (described below) used in each lane are as follows: (Lane 1) CAST/Ei; (lane 2) $\mathrm{DBA} / 2 \mathrm{l}$; (lane 3) C3HeB/Fel; (lane 4) C57BL/6); (lane 5) B6CBACa-A $\mathrm{A}^{\mathrm{w}-1} / \mathrm{A}$; (lane 6) $L C$ heterozygote $(\mathrm{LC} /+$ ); (lane 7) LC/cast. F1 heterozygote; (lane 8) CL103; (lanes 9,10) CL222, (lane 11), a nonrecombinant sample from Lc/cast. backcross with only M. m. musculus background; (lane 12) CL230; (lane 13) CL340; (lane 14) CL369; (lane 15) CL391. Approximately $100 \mathrm{ng}$ of genomic DNA was amplified in each lane. (B)Summary of the genotyping of six recombinant animals from the $L c /$ cast. backcross with three gene markers and eight MIT markers in the $L C$ region on Chromosome 6 . The six recombinant animals from the $L C / c a s t$. backcross are labeled CL222, CL340, CL230, CL103, CL369, and CL391, and their phenotypic status is indicated beneath their names in each column. Both nonrecombinant and recombinant chromosomes are illustrated for each animal. $M . m$. musculus includes one of four possible alleles: $\mathrm{C} 57 \mathrm{BL} / 6 \mathrm{~b}, \mathrm{DBA} / 2 \mathrm{~J}, \mathrm{C} 3 \mathrm{HeB} / \mathrm{Fe}$, and $\mathrm{CBA} / \mathrm{CaCnLe}$, all of which may be present in the vicinity of the $L C$ locus. The order of markers telomeric to $L C$ cannot be determined in this typing. The markers used and polymorphisms detected are summarized in Table 1 and described in Methods. The relative order of D6Mit123 and D6Mit 122 was determined later in the text. The location of the Lc mutation can be derived from the typing of these recombinant animals as follows: Provided that in this backcross only the recombinant chromosomes could carry the $L c$ mutation, and that their affected status is independently determined, we reasoned that CL222, CL340, and CL230 must have inherited the mutation in their M. m. musculus segments. Thus, $L C$ lies telomeric to D6Mit121. Similarly CL391 must have inherited the mutation in its $M . m$. musculus segment, placing LC centromeric to D6Mit175. Conversely CL103 and CL369 must have inherited the wild-type gene in their M. m. castaneus segment, indicating that $L c$ is centromeric to D6Mit175.

important recombinants centromeric to $L c$ (CL222, CL230, and CL340) do resolve the four centromeric markers, D6Mit123, D6Mit122, D6Mit94, and D6Mit121, with marker D6Mit121 being closest to $L c$. However, the other three recombinants telomeric to Lc (CL103, CL369, and CL391) do not resolve the four telomeric markers D6Mit175, D6Mit174, D6Mit95, and D6Mit162.

To determine the closest marker telomeric to $L c$ and to confirm the order of the centromeric markers, we decided to use a generic backcross with a different strain background. In theory this backcross should provide additional recombina- tion events within the $L c$ region as well as a different distribution of crossover events. We utilized the EUCIB because it is a backcross with a total number of 982 meioses between $\mathrm{C} 57 \mathrm{BL} / 6$ and M. spretus (The European Backcross Collaborative Group 1994). For convenience, only 580 DNA samples from the London collection (a subset of the 982 DNA samples) were analyzed in this study. To minimize the total amount of DNA typing, we chose only a small set of DNA samples, 184, that have known crossovers between the two closest Chromosome 6 landmarks, D6Nds4 and D6Nds5. These markers are separated by a 
ZUO EI AL.

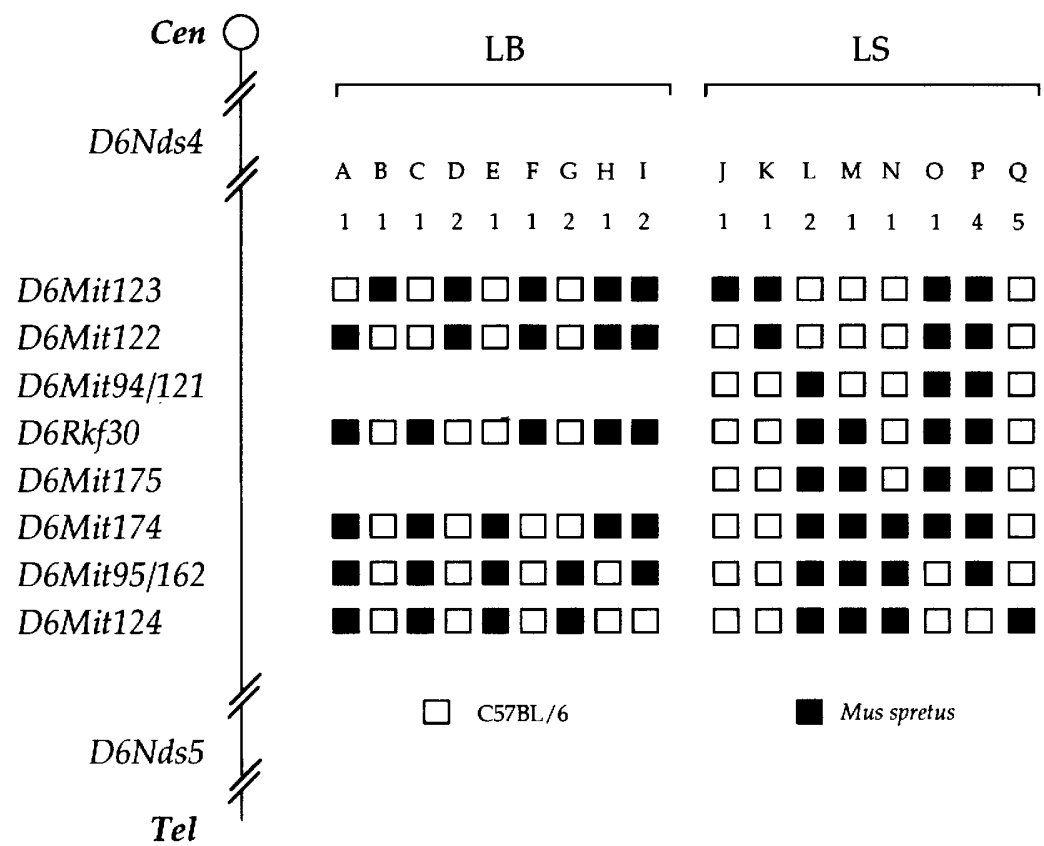

Figure 2 Genotyping of 28 DNA samples from EUCIB with nine MIT markers and a novel YAC end probe in the $L C$ region on Chromosome 6. (LB)Samples from the backcross with the C57BL/6 parental strain; (LS) the complementary backcross with the $M$. spretus parental strain. A total of 17 types of recombinant chromosomes (A-Q) from 28 samples are illustrated; the number beneath each letter $(A-Q)$ indicates the number of individuals displaying that type of recombinant chromosome. (A) LB437; (B) LB596; (C) LB159; (D) LB346 and LB409; $(E)$ LB158; ( $F$ LB532; (G) LB205 and LB208; (H) LB206; ( $)$ LB202 and LB420; (N) LS786; (K) LS869; (L) LS216 and LS769; (M) LS315; (N) LS702; (O) LS588; (P) LS181, LS191, LS377, and LS830; (Q) LS189, LS193, LS378, LS766, and LS872. The typing results of LB samples with D6Mit94, D6Mit121, and D6Mit175 are ambiguous, because these markers failed to amplify the $M$. spretus alleles at these loci. Markers are summarized in Table 1 and described in the text; the novel marker D6Rkf30 is described later in the text.

genetic distance of $\sim 30 \mathrm{cM}$ and flank the $L c$ locus (The European Backcross Collaborative Group 1994). Based on the known map of the MIT markers, we then typed these 184 samples with two MIT markers, D6Mit123 and D6Mit124, that flank the $L c$ locus and are separated by a relatively large distance. The typing resulted in a total of $28 \mathrm{sam}$ ples that have recombination events between D6Mit123 and D6Mit124. We then typed these 28 samples with the other seven MIT markers in the $L c$ region to determine the relative order of the markers (Fig. 2). These results confirmed that D6Mit121 is the closest marker to $L c$ on the centromeric side. More importantly, D6Mit175 was found to be the closest marker to $L c$ on the telomeric side. D6Mit121 and D6Mit175 are sepa- rated by a total of five recombinants in 1084 meiosis (four out of 504 in the $L c /$ cast. backcross and one out of 580 in the EUCIB), indicating a relatively small genetic distance of $0.5 \mathrm{cM}$.

\section{Construction of a YAC Contig Spanning the LC Locus}

Using the two closest markers that flank the $L c$ locus D6Mit121 and D6Mit175, we screened a mouse YAC library to begin chromosomal walking. From the centromeric side of $L c$, we obtained three YACs containing D6Mit121 (6A12, 23E5 and 140F10), whereas from the telomeric side, three other YACs were obtained containing D6Mit175 (171F8, $2 \mathrm{H} 6$, and 102E8). In addition, we identified four other YACs (37F2, $7 \mathrm{G8}, 55 \mathrm{H} 7$, and 24C8) at the D6Rkf39 locus. We further characterized these 10 YACs by rescuing their end sequences, determining their insert sizes and analyzing their content of markers (Table 1).

A total of 15 YAC ends were isolated by a combination of the inverse PCR and homologous recombination methods (Hermanson et al. 1991; Silverman et al. 1991; Zuo et al. 1992). For each YAC end isolated, we first confirmed that it was from Chromosome 6 by using either a panel of somatic cell hybrid (SCH) cell lines (Fig. 3), or a panel of these 10 YACs isolated from this region and described above. By these analyses, we found that, among the 15 YAC ends isolated, 10 are from Chromosome 6 (Table 1) and two are from regions other than Chromosome 6 (J. Zuo, P. De Jager, and N. Heintz, unpubl.). In addition, two other YAC ends are repetitive, and one remains uncharacterized. All three, therefore, have not been assigned to Chromosome 6 (described later in Fig. 6).

To confirm their close linkage to the $L c$ locus, we utilized important polymorphic portions of these YAC end probes (D6Rkf15, D6Rkf29, and $D 6 R k f 30$ ) to type the 21 recombinant animals near the $L c$ locus, including the 6 important recombinants between D6Rkf41 and D6Rkf33/ $D 6 R k f 39$ (Fig. 4 and Table 1). The YAC end probe, 
Table 1. Summary of the genetic and physical mapping of the $L c$ region

\begin{tabular}{|c|c|c|c|c|c|c|c|c|c|c|c|c|c|c|c|c|c|c|c|}
\hline & & & & & $\begin{array}{c}\text { YAC } \\
\text { Size (kb) }\end{array}$ & $\begin{array}{c}119 G 7 \\
700\end{array}$ & $\begin{array}{l}6 A 12 \\
450\end{array}$ & $\begin{array}{l}23 \text { E5 } \\
250\end{array}$ & $\begin{array}{c}140 \mathrm{~F} 10 \\
200\end{array}$ & $\begin{array}{c}157 \mathrm{G1} \\
700\end{array}$ & $\begin{array}{c}183 \mathrm{E} 6 \\
650\end{array}$ & $\begin{array}{l}161 F 7 \\
620\end{array}$ & $\begin{array}{c}171 \mathrm{~F} \\
900\end{array}$ & $\begin{array}{l}2 \mathrm{H} 6 \\
800\end{array}$ & $\begin{array}{c}102 \mathrm{~EB} \\
500\end{array}$ & $\begin{array}{l}\mathbf{3 7 F 2} \\
1000\end{array}$ & $\begin{array}{l}7 \mathrm{G} 8 \\
480\end{array}$ & $\begin{array}{l}55 \mathrm{H} 7 \\
920\end{array}$ & $\begin{array}{l}24 C 8 \\
600\end{array}$ \\
\hline Marker & Source & Bin & Polymorphism & Somat. & RI/D3(kb) & & & & & & & & & & & & & & \\
\hline D6Rkg1 & E5 end clone & & & chr.6 & 21 & $\mathbf{x}$ & $x$ & $\mathbf{x}$ & & & & & & & & & & & \\
\hline D6Rlg2 & E5 subclone & I. & & & $1.3 / 4$ & & $x$ & $x$ & & & & & & & & & & & \\
\hline D6R\&B & E5 subcione & I & & & $4.6 / 5.5$ & & $\mathbf{x}$ & $x$ & & & & & & & & & & & \\
\hline D6Mit121 & MTT marker & II & SSL.P & & & & $\mathbf{x}$ & $x$ & $\mathbf{x}$ & & & & & & & & & & \\
\hline D6RLf4 & E5 subclone & II & & & $0.45 / 6$ & & $x$ & $x$ & $\mathbf{x}$ & & & & & & & & & & \\
\hline D6R 45 & E5 subclone & II & & & $21 /$ & & $\mathbf{x}$ & $\mathbf{x}$ & $x$ & & & & & & & & & & \\
\hline D6RLf6 & E5 subclone & m & & & $2.8 / 5.8$ & & & $\mathbf{x}$ & & & $x$ & $\mathbf{x}$ & & & & & & & \\
\hline D6RLG & E6 subclone & m & & & 21 & & & $x$ & & & $\mathbf{x}$ & $\mathbf{x}$ & & & & & & & \\
\hline DGRlges & E6 subclone & III & & & $15 / 4.1$ & & & $\mathbf{x}$ & & & $\mathbf{x}$ & $x$ & & & & & & & \\
\hline D6RK\& & E6 subclone & III & & & $2.9 / 3.5$ & & & $x$ & & & $x$ & $x$ & & & & & & & \\
\hline D6R\&f10 & E6 subclone & III & & & $2.8 / 2.5$ & & & $\mathbf{x}$ & & & $x$ & $\mathbf{x}$ & & & & & & & \\
\hline D6Rkf11 & E6 subclone & III & & & $1.7 / 1$ & & & $\mathbf{x}$ & & & $x$ & $x$ & & & & & & & \\
\hline D6RKf12 & E6 subclone & III & & & $2.6 / 2.7$ & & & $\mathbf{x}$ & & & $\mathbf{x}$ & $x$ & & & & & & & \\
\hline$D 6 R \& 73$ & E6 subclone & III & & & $2.7 / 6.5$ & & & $x$ & & & $x$ & $\mathbf{x}$ & & & & & & & \\
\hline D6Rlg14 & IRS-PCR & III & SSLP & & 71 & & & $\mathbf{x}$ & & & $\mathbf{x}$ & $x$ & & & & & & & \\
\hline D6Rlf15 & E5 end clone & IV & RFLP(Hae III) & chr.6 & 4.61 & & & $\mathbf{x}$ & & $\mathbf{x}$ & $\mathbf{x}$ & $x$ & & & & & & & \\
\hline D6R\&A16 & Es subclone & $v$ & & & $1.6 / 2.9$ & & & & & $x$ & $x$ & $\mathbf{x}$ & & & & & & & \\
\hline D6Rlg17 & E6 subclone & $\mathrm{v}$ & SSCP & & $18 / 1.7$ & & & & & $x$ & $x$ & $\mathbf{x}$ & & & & & & & \\
\hline D6Rkg18 & Gl subcione & $\mathrm{v}$ & & & $0.9 / 28$ & & & & & $\mathbf{x}$ & $\mathbf{x}$ & $x$ & & & & & & & \\
\hline D6Rlg19 & G1 subcione & $\mathrm{v}$ & & & $1.2 / 10$ & & & & & $x$ & $\mathbf{x}$ & $x$ & & & & & & & \\
\hline D6Rlg20 & G1 subclone & $\mathrm{v}$ & & & $0.7 / 2.8$ & & & & & $x$ & $\mathbf{x}$ & $\mathbf{x}$ & & & & & & & \\
\hline D6R\& 21 & G1 subclone & $\mathrm{v}$ & & & $0.45 / 3$ & & & & & $\mathbf{x}$ & $\mathbf{x}$ & $x$ & & & & & & & \\
\hline D6Rk 22 & G1 subclone & $\mathrm{v}$ & & & $0.5 / 11$ & & & & & $x$ & $x$ & $x$ & & & & & & & \\
\hline$D 6 R \times 23$ & G1 subclone & $\mathrm{v}$ & & & $0.5 / 7.5$ & & & & & $x$ & $x$ & $\mathbf{x}$ & & & & & & & \\
\hline$D 6 R \& 24$ & G1 subclone & $\mathrm{v}$ & RFLP(Pst I) & & $1.4 / 11$ & & & & & $x$ & * & $x$ & & & & & & & \\
\hline$D 6 R k g 25$ & E6 subclone & VI & $\operatorname{SSCP}$ & & $1.2 / 2.6$ & & & & & $x$ & $x$ & & & & & & & & \\
\hline D6RKg26 & G1 subclone & VI & & & $2.9 / 4$ & & & & & $x$ & $\mathbf{x}$ & & & & & & & & \\
\hline D6Rkg27 & G1 subclone & VII & & & $0.8 / 7.5$ & & & & & $x$ & & & & & & & & & \\
\hline D6Rkg28 & G1 subcione & VII & & & $2.2 / 4.8$ & & & & & $\mathbf{x}$ & & & & & & & & & \\
\hline$D 6 R+29$ & H6 end clone & VIII & $\operatorname{SSCP}$ & chr.6 & $3.8 /$ & & & & & $x$ & & & $x$ & $x$ & & & & & \\
\hline D6R\&30 & EB end clone & Dx & Novel & chr. 6 & 8.81 & & & & & $x$ & & & $x$ & $x$ & $x$ & & & & \\
\hline D6Rlg31 & G1 subclone & D & & & $1.4 / 11$ & & & & & $\mathbf{x}$ & & & $\mathbf{x}$ & $\mathbf{x}$ & $\mathbf{x}$ & & & & \\
\hline$D 6 R k \beta 32$ & IRS-PCR & $\mathrm{IX}$ & & & & & & & & $\mathbf{x}$ & & & $\mathbf{x}$ & $x$ & $x$ & & & & \\
\hline D6Mil175 & MIT marker & & SSLP & & & & & & & & & & $\mathbf{x}$ & $x$ & $x$ & & & & \\
\hline$D 6 R \& f 33$ & Gene marker & & RFLP(Rsa I) & chr. 6 & & & & & & & & & $x$ & $x$ & $x$ & & & & \\
\hline D6Mit174 & MIT marker & & SSLP & & & & & & & & & & $x$ & $x$ & $x$ & & & & \\
\hline D6Rł $\Varangle 34$ & F2 end clone & & & chr. 6 & $4 /$ & & & & & & & & $x$ & $x$ & & $x$ & & & \\
\hline D6Mil162 & MIT marker & & SSLP & & & & & & & & & & & $x$ & & $x$ & & & \\
\hline$D 6 R k 635$ & G8 end clone & & & & $5 /$ & & & & & & & & & * & & $x$ & $x$ & & \\
\hline D6Rlg36 & H7 end clone & & & & & & & & & & & & & * & & $\mathbf{x}$ & $x$ & $x$ & \\
\hline D6Rlf97 & H6 end clone & & & & $1.1 /$ & & & & & & & & & $x$ & & $x$ & $x$ & $x$ & \\
\hline D6Rk338 & OS end clone & & & & 41 & & & & & & & & & & & $x$ & $x$ & $x$ & $x$ \\
\hline D6Rł39 & Gene marker & & RFLP(Rsa I) & chr. 6 & & & & & & & & & & & & $x$ & $x$ & $x$ & $x$ \\
\hline$D 6 R k 440$ & F2 end clone & & & chr.6 & 2.81 & & & & & & & & & & & $x$ & & $\mathbf{x}$ & $\mathbf{x}$ \\
\hline
\end{tabular}

All markers used in this study are listed in the first column. The second column indicates their source. In the third column, markers that hybridize to the same set of overlapping YACs are grouped together to form nine bins, I to IX. The fourth column summarizes polymorphisms that have been tested on the $L C /$ cast. backcross (see Methods). The fifth column indicates the markers that have been mapped to Chromosome 6 by somatic cell hybrid mapping analysis. The sixth column indicates the size (in kb) of EcoRI (RI) and HindIII (D3) genomic fragments to which the markers hybridize. The top rows indicate the names and the insert sizes of the 14 YACs used in this study. Their probe content is as follows: $(x)$ Positive signals by both Southern blot hybridization and PCR analysis; $\left({ }^{*}\right)$ failure to detect positive signal by Southern blot hybridization or PCR amplification, possibly because of a rearrangement within the YAC. 
ZUO EI AL.

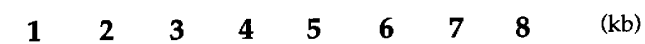

A

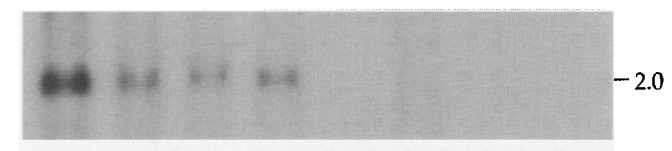

B

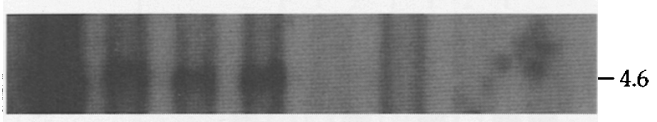

C
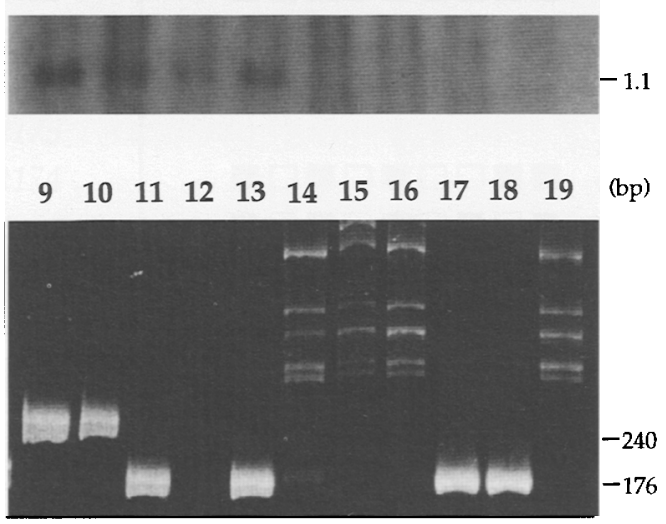

Figure 3 Examples of the localization of YAC end probes on mouse Chromosome 6 using a somatic cell hybrid mapping panel. ( $A-C$ ) Autoradiographs of Southern blots made from $\mathrm{SCH}$ lines. The probes used in A-CYAC end probes, D6Rkf1, D6Rkf15, and $D 6 R k f 37$, respectively. Approximately $5-10 \mu \mathrm{g}$ of genomic DNA was digested with EcoRI and loaded into each lane in $A-C$. (D) PCR amplification from $\sim 100 \mathrm{ng}$ of genomic DNA with primers derived from YAC end probe D6Rkf30. The DNA samples are as follows: (Lane 1) $L C /$ +heterozygote; (lanes 2,13) 2A2B1; (lane 3,17) 2A2H3; (lanes 4,18) BEM1-4; (lanes 5,16) E36; (lanes 6,15) 2A2C2; (lanes 7,19) ECM4C; (lanes 8,14) R2-24; (lane 11) C57BL/6); (lane 10) CAST/Ei; (lane 9) SPRET/Ei; (lane 12) control without DNA. Positive signals in hybrids $2 \mathrm{~A} 2 \mathrm{~B} 1$, 2A2H3, and BEM1-4 (lanes 2,3,4,13,17,18) as well as in genomic DNA (lanes $1,9,10,11$ ) in the absence of signal in any other hybrid lines indicate a Chromosome 6 location.

$D 6 R k f 15$, is one recombination event centromeric to $L c$ and nonrecombinant with D6Mit121; the other two YAC ends, D6Rkf29 and D6Rkf3o, are three recombination events telomeric to $L c$ and nonrecombinant with D6Mit175. Although these polymorphic YAC end probes have not crossed any of the four crucial recombination breakpoints, they do provide closer flanking markers which refine the $L c$ locus.

Using two of these YAC end probes, D6Rkf15 and $D 6 R k f 30$, we screened the YAC library again and identified a common YAC, 157G1, containing both probes. In addition, two other YACs, 188E6 and 161F7, were identified from D6Rkf15, and another YAC, 119G7, was isolated from D6Rkf1. We therefore have identified a total of 14 YACs that span a large genomic interval, from $D 6 R k f 1$ to $D 6 R k f 40$, in the vicinity of the $L c \mathrm{mu}$ tation (Table 1). The $L c$ locus, as defined by the two flanking markers D6Rkf15 and D6Rkf3O is spanned by five YACs with threefold redundancy in most regions. A single YAC with a $700-\mathrm{kb}$ insert, $157 \mathrm{G} 1$, spans the entire $L c$ candidate region between markers D6Rkf15 and D6Rkf30.

\section{Subcloning YACs to Obtain High Density Markers from the $L c$ Region}

To refine the genetic and physical maps of the $L c$ locus, we chose to subclone our YAC DNA into a smaller and more convenient cloning system to obtain more markers. Sublibraries were made from YACs $157 \mathrm{G} 1,188 \mathrm{E} 6$, and 23E5 (see Methods), and a total of 26 plasmid clones were mapped to the YAC contig spanning the $L c$ locus. These subcloned probes, in addition to YAC end probes, gene markers, and MIT markers, can be further categorized into groups or "bins" (Zuo et al. 1993). Each bin consists of markers that hybridize to the same set of YACs (Table 1). An example of such a hybridization with four probes is shown in Figure 5. In this analysis, we also found that nine out of 19 subcloned probes from YAC 188E6 are not from Chromosome 6, suggesting that this YAC is chimeric. Similarly, as all 11 subcloned probes isolated from YAC 157G1 (bin IVIX in Table 1) are mapped to Chromosome 6, we assumed that the YAC 157G1 is a nonchimeric YAC.

Some of these subcloned probes were developed into polymorphic markers to refine the genetic map of the $L c$ locus. D6Rkf17 and D6Rkf24 in bin V, and D6Rkf25 in bin VI, were such polymorphic markers and were utilized in genetic typing of the 21 recombinant animals in the $L c$ region as described above (Fig. 4). In addition, interspersed repetitive sequence PCR was used to isolate additional internal probes from these YACs, such as the polymorphic marker D6Rkf14 (Fig. 4; Table 1). Combining the genetic mapping and YAC hybridization binning data, the $L c$ locus was refined and now consists of bins VI and VII between D6Rkf25 and D6Rkf29, the two closest flanking markers (Fig. 6). 


\section{A}
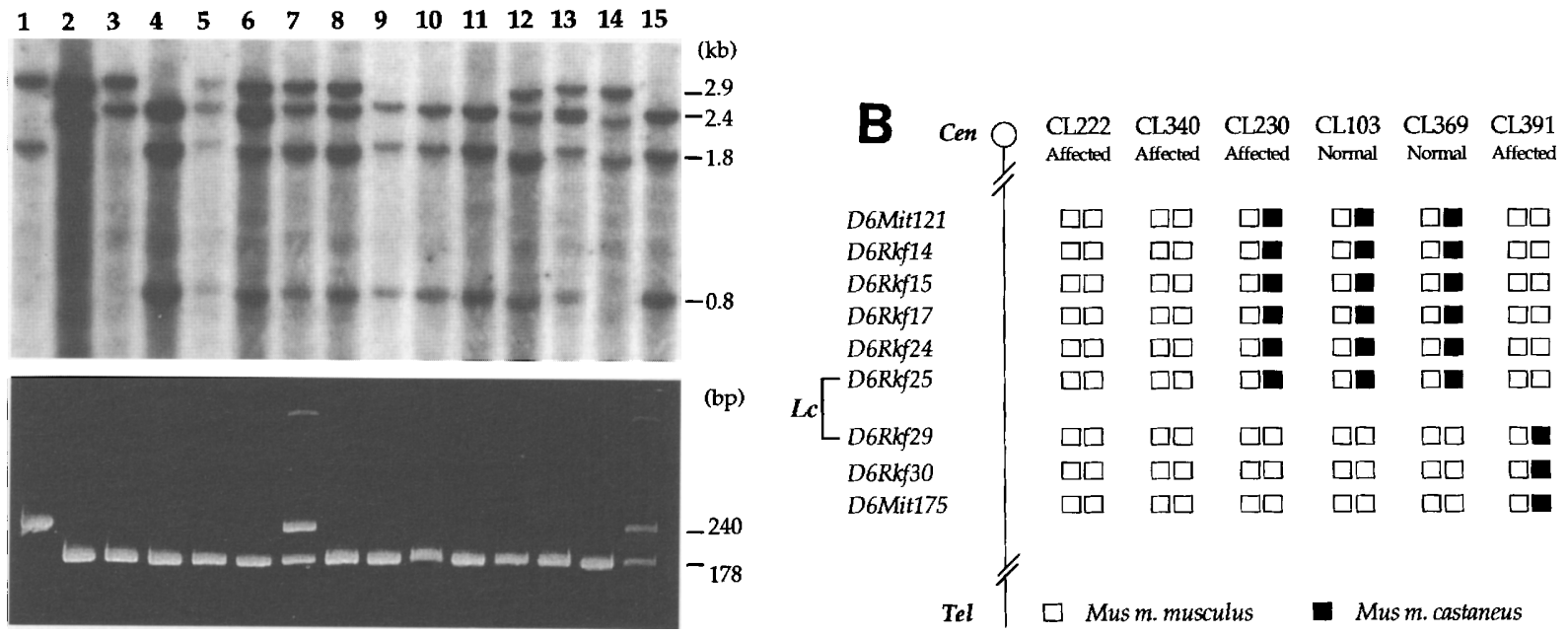

Figure 4 Genotyping of the six recombinant animals from the $L C / c a s t$. backcross with markers within a refined $L C$ region on Chromosome 6. DNA samples used in $A$ are the same as described in Fig. 1A. (A) (top) Autoradiograph of a genomic Southern blot hybridized with the YAC end probe D6Rkf15. Approximately 5-10 $\mu \mathrm{g}$ of genomic DNA was digested with Haell and loaded into each lane. D6Rkf15 detects three different alleles: a $M$. m. castaneus allele of 2.9-kb and 1.8-kb fragments, a C57BL/6) allele of 2.4-, 1.8- and 0.8-kb fragments, and a common allele between $\mathrm{DBA} / 2 \mathrm{~J}, \mathrm{C} 3 \mathrm{HeB} / \mathrm{Fe}$, and $\mathrm{CBA} / \mathrm{CaCnLe}$ of 2.9- and 2.4-kb fragments. (Bottom) The products of PCR amplification with the primer pair derived from the polymorphic YAC end marker D6Rkf30. Approximately $100 \mathrm{ng}$ of genomic DNA was used for amplification in each lane. (B) Summary of the genotyping of the six recombinant animals in the $L C /$ cast. backcross with polymorphic markers that map between D6Mit 121 and D6Mit175. These markers include YAC end probes and YAC subclones and their order was determined as described later in the text. The six recombinant animals and their other features are as described in Fig. 1B. None of the polymorphic markers from the $L C$ region, except $D 6 R k f 15$, were able to distinguish definitively among the four inbred strain alleles, DBA/2J, C3HeB/Fel, CBA/CaGnLe, and C57BL/6).

\section{DISCUSSION}

As an important step towards the isolation of the $L c$ gene, we report here the construction of a high resolution genetic map and a YAC contig with a high density of markers in the $L c$ locus.

The YAC contig reported here consists of 14 YACs with an average size of $630 \mathrm{~kb}$ and a threefold redundancy. The most centromeric and telomeric markers of this contig, D6Rkf1 and D6Rkf40, have been confirmed to be from Chromosome 6 by SCH mapping analysis. Evenly distributed within this contig, a large number of markers have been extensively characterized by a combination of SCH mapping analysis, genetic linkage analysis, and YAC content mapping. This set of independent analyses are consistent with each other and therefore confirm the continuity of the YAC contig. Although YAC 157G1 has a small internal segment (bin VII) not contained in any other redundant YAC clones, it is nevertheless likely to be colinear with genomic DNA;
D6Rkf15 and D6Rkf30, which both map to this $700-\mathrm{kb}$ YAC, also share one $600-\mathrm{kb}$ Sall fragment and another 1-Mb partial Sall fragment in genomic pulsed-field gel electrolphoresis (PFGE) analysis (J. Zuo, P. De Jager, and N. Heintz, unpubl.).

Based on the sizes of the nonchimeric YACs, their overlapping pattern, and the physical distances between markers, we estimate that the distance between D6Rkf1 and D6Rkf40 is $\sim 2.6 \mathrm{Mb}$. With additional YACs isolated from D6Rkf1 and D6Rkf40, our YAC contig spans $>3 \mathrm{Mb}$ of genomic DNA. Assuming a random distribution of the subcloned probes over a YAC, we were able to refine the physical distances between these markers. The Chromosome 6 portion (bin III-VI in Table 1) of $650-\mathrm{kb}$ YAC $188 \mathrm{E} 6$, for example, is estimated to be $-310 \mathrm{~kb}$ as 10 of 19 subcloned probes are mapped within bin III-bin VI of this contig. Similarly, among 11 157G1 subclones that are mapped to this contig, three are within bins VI and VII. Based on the 700-kb size of the 157G1 YAC, the estimated size of bins VI and VII 


\section{ZUO ET AL.}

is $-300 \mathrm{~kb}$. Therefore, the minimal candidate region of the $L c$ locus is further defined by the two closest flanking markers, D6Rkf25 and D6Rkf29, which are contained within a genomic segment of $\sim 300 \mathrm{~kb}$. This refined physical map will greatly facilitate the establishment of a P1 or BAC contig of the $L c$ region and the analysis of candidate genes isolated from this relatively small genomic region.

In the $L c$ phenotypic backcross between B6CBACa- $\mathrm{A}^{\mathrm{w}-\mathrm{J}} / \mathrm{A}-\mathrm{L} c$ and $M . m$. castaneus, there are a total of four recombination events out of 504 meioses in the $L c$ region smaller than $600 \mathrm{~kb}$. In the EUCIB backcross between $M$. spretus and $\mathrm{C} 57 \mathrm{BL} / 6$, however, the crossovers seem to be distributed evenly in this region of the chromosome. Statisitical analyses suggest that these two recombination frequencies in the $L c$ region (4/ 504 and $1 / 580$ ) are not significantly different $(P>0.25, \mathrm{df}=1)$ from the mean value $(5 / 1084)$ (Reeves et al 1990). In addition, we have not found any differences between wild-type and $L c /$ + genomic DNA in the long range restriction maps of the region (J. Zuo et al., unpubl.). Therefore, the $L c$ mutation is unlikely to involve any gross genomic rearrangements.

Many approaches have been described for the recovery of polymorphic probes from YACs (Chen et al. 1995). The strategy we used here is simple and efficient. It consists of subcloning YAC DNA into the $\lambda$-Zap phagemid followed by direct PCR amplification of recombinant subcloned fragments. These PCR products can be directly sequenced and further developed into sequence-tagged sites (STSs). Furthermore, most of these STSs can be easily used as polymorphic markers by single-strand confirmation polymorphism (SSCP). Finally, these clones can be directly used as hybridization probes, to identify conserved sequences by "zooblot", or to screen P1 or BAC libraries.

Given the progress now evident in the mouse genome project, it seems reasonable that the approach we have taken to establishing genetic and physical maps of mutant loci can be broadly applied. The construction of a high resolution genetic map of a desired locus can be established using the available MIT polymorphic markers on a generic backcross such as EUCIB. Simultaneously, one or two phenotypic crosses specific for the desired mutant can be typed with these same MIT markers. The use of one or two subspecific strains different from those involved in the generic cross will ensure the even distribution of informative crossovers. In addition to a comparative map of the region of interest, these phenotypic crosses will order the markers that flank the mutant locus. The two closest flanking polymorphic markers can then be used to type pups before they are weaned, so that only those pups with informative crossovers will be kept for later phenotypic studies. This measure can significantly decrease the cost of maintenance of large numbers of offspring. It is after this step that YACs should be isolated from the two closest flanking markers, and a YAC contig can then be constructed with minimal effort. With the availability of MIT markers and EUCIB DNA samples, the initial genetic analysis necessary for physical mapping can be made significantly more efficient.

\section{METHODS}

\section{DNA Markers and Sequences}

Two known gene markers, Cbl-1 (D6Rkf41) and Etl-1 (D6Rkf33), were described previously (Norman et al. 1991, 1995). A third gene marker, Jrl (D6Rkf39), is an 880-bp genomic fragment isolated from pJrl-1, kindly provided by Dr. J. R. Hansbrough (Washington University, St. Louis, MO). MIT markers or Map-pairs were purchased through Research Genetics, Inc. Novel PCR primers and markers used in this study are listed in Table 1 and 2.

\section{Southern Blot Hybridization}

All probes were labeled by random priming. Hybridization was performed using standard procedures at $65^{\circ} \mathrm{C}$ in a Hybridization Incubator (Robbins Scientific model 400) and filters were then washed at $65^{\circ} \mathrm{C}$ in $0.2 \times \mathrm{SSC}$ and $0.1 \%$ SDS for $2 \times 30 \mathrm{~min}$. Finally, XAR Kodak films were exposed to the filters at $-70^{\circ} \mathrm{C}$ for $24-72 \mathrm{hr}$.

\section{Sequencing and Homology Searches}

DNA sequencing was performed on either $\mathrm{ABI} 370 \mathrm{~A}$ or 373A automated sequencers (Applied Biosystems, Inc.) using both PCR products and plasmids as templates, or by manual procedures using plasmids as templates. Sequence homology searches were performed using the National Center for Biotechnology Information (NCBI) data base.

\section{PCR Amplification}

DNA Thermal Cycler, GeneAmp PCR System 9600 (PerkinElmer Cetus) and DNA Engine (M.J. Research, Inc.) were used for PCR amplification in this study. Four different buffer conditions were used: TNK25, TNK50, TNK100 (Zuo et al. 1992) and $10 \times$ PCR buffer (Perkin-Elmer Cetus). All PCR reactions were carried out in $25-\mu$ l volume. Samples were processed through an initial denaturation $\left(94^{\circ} \mathrm{C}\right.$ for 4 


\section{A YAC CONTIG OF THE LURCHER LOCUS}

$\begin{array}{lllllllllll}1 & 2 & 3 & 4 & 5 & 6 & 7 & 8 & 9 & 10 & (\mathrm{~kb})\end{array}$

A

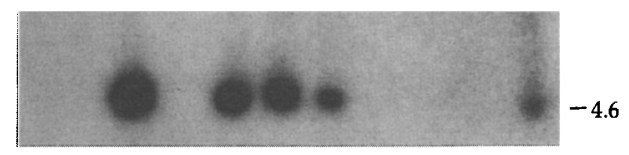

B

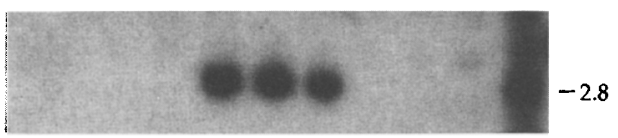

C

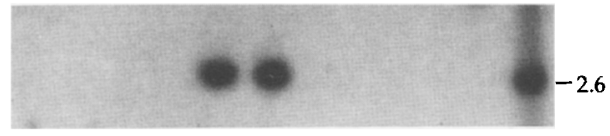

D

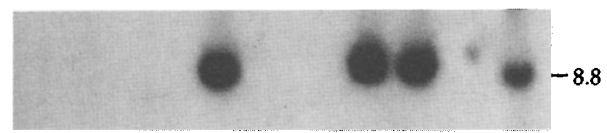

Figure 5 Overlap of YAC clones by Southern blot hybridization analyses with four DNA markers in the refined $L C$ region between D6Mit 121 and D6Mit 175 on Chromosome 6. A Southern blot of ECoRIdigested DNA from nine YACs in the $L c$ region, as well as mouse genomic DNA, was sequentially hybridized with two YAC end probes, D6Rkf15 $(A)$ and $D 6 R k f 30(D)$. Another Southern blot of the same DNA samples digested with HindIII was sequentially hybridized with two subcloned probes, D6Rkf18 $(B)$ and D6Rkf25 (C). (Lane 1) YAC 6A12; (lane 2) YAC 23E5; (lane 3) YAC 140F10; (lane 4) YAC 157G1; (lane 5) YAC 188E6; (lane 6) YAC 161F7; (lane 7) YAC 171F8; (lane 8) YAC 2H6; (lane 9) ADH-G1, a YAC isolated from D6Rkf41; (lane 10) B6CBACa$A^{\mathrm{w}-1} / \mathrm{A}$ genomic DNA. YAC agarose blocks with $\sim 1 \times 10^{7}$ cells/block, and genomic agarose blocks with $\sim 1 \times 10^{6}$ cells/block were digested and analyzed in each lane.

$\min )$, then 35 cycles of denaturation $\left(94^{\circ} \mathrm{C}, 1 \mathrm{~min}\right)$, annealing $(1 \mathrm{~min})$, and elongation $\left(72^{\circ} \mathrm{C}, 3 \mathrm{~min}\right)$, followed by $10 \mathrm{~min}$ elongation at $72^{\circ} \mathrm{C}$ and stored at $4{ }^{\circ} \mathrm{C}$. The annealing temperature for each PCR primer pair is listed in Table 2 .

\section{YAC Isolation and Characterization}

The YAC library purchased from Research Genetics, Inc. was screened by PCR using standard procedures. Positive clones were purified; both agarose blocks $\left(5 \times 10^{7}\right.$ cells/ block) and liquid DNA were made as described previously (Zuo et al. 1992). The sizes of YAC inserts were determined by PFGE as described below. YAC agarose blocks were digested according to the protocol from New England Biolabs and analyzed by regular gel electrophoresis, or PFGE in a Bio-Rad CHEF DRII apparatus in $0.5 \times \mathrm{TBE}$ at $14^{\circ} \mathrm{C}$ at $6 \mathrm{~V} / \mathrm{cm}$ for $24-36 \mathrm{hr}$ under conditions to fractionate DNA from 50 to $1500 \mathrm{~kb}$. Gels were then acid-nicked and transfered to GeneScreenPlus or Hybond- $\mathrm{N}^{+}$membranes (Amersham). Both the inverse PCR and homologous recombination methods were used to rescue the end sequences of
YACs; procedures and sequences have been described elsewhere (Hermanson et al. 1991; Silverman et al. 1991; Zuo et al. 1992).

Mice

Mice used in this study were purchased from the Jackson Laboratory and maintained at the Specific Pathogen Free

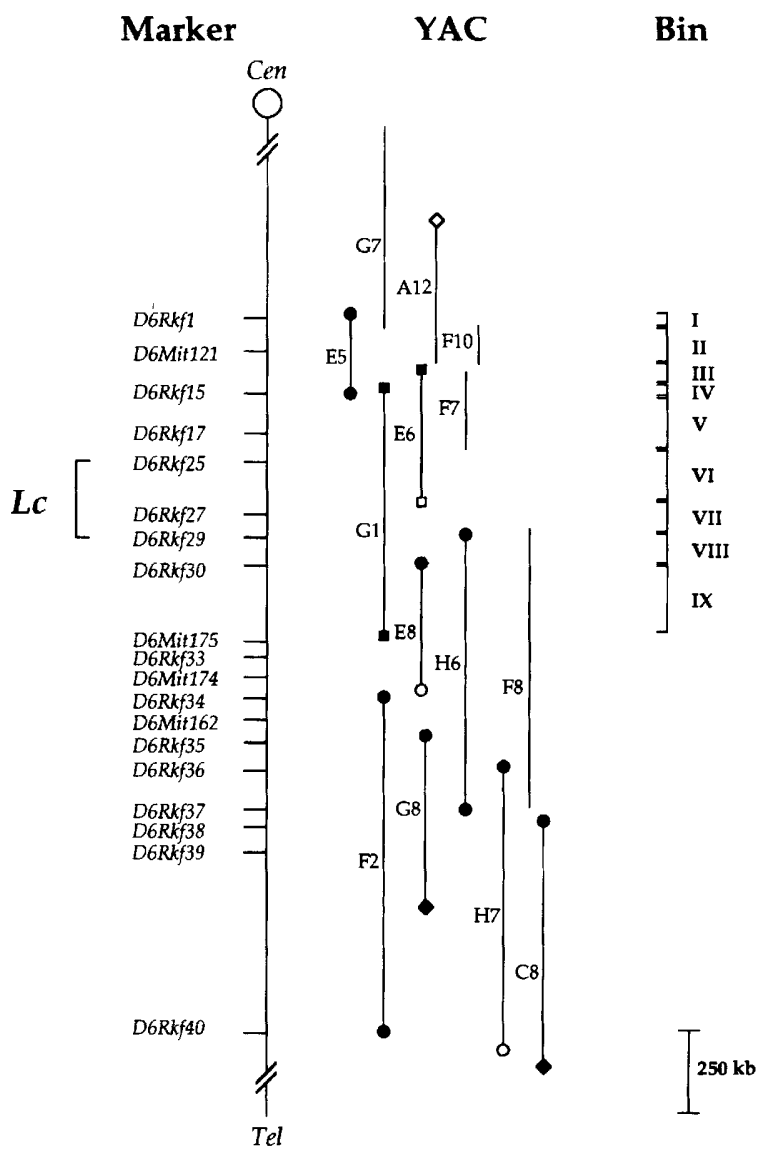

Figure 6 A composite map of the $L C$ region on Chromosome 6. The markers presented here are described in Table 1 . One marker from each bin is included in this diagram. The 14 YACs are labeled with their abbreviated names. (O) Nonchimeric Chromosome 6 YAC ends; (O) chimeric YAC ends; (D) assumed to be nonchimeric Chromosome 6 YAC ends; ( $\square$ ) assumed to be chimeric YAC ends; $(\diamond)$ repetitive YAC ends; $(\diamond)$ YAC ends that have not been characterized. Based on the meiotic recombination events, the sizes of nonchimeric YACs, their overlapping pattern, estimation of the sizes of bins, and physical distances between markers, these 14 YACs can be assembled into a contig and the order of markers can be determined. The contig starts from D6Rkf1 and ends at D6Rkf40, covering $\sim 3 \mathrm{Mb}$ of genomic DNA that contains the $L C$ locus. The $L C$ mutation is mapped between the two closest polymorphic markers, D6Rkf25 and D6Rkf29; this region is estimated to be $300 \mathrm{~kb}$ in size (see Discussion). 
Table 2. Sequences of primers used in this study and their PCR conditions

\begin{tabular}{|c|c|c|c|}
\hline Marker & Primer pair & $\begin{array}{l}\text { Anneal. } \\
\text { temp }\left({ }^{\circ} \mathrm{C}\right)\end{array}$ & $\begin{array}{l}\text { Product } \\
\text { size (bp) }\end{array}$ \\
\hline \multirow[t]{2}{*}{$D 6 R k f 1$} & 1: 5'-ATC TGC CTT TAC ACA TTG CC-3' & 55 & 245 \\
\hline & 2: 5'-TTC TCT AGT GAT GCA AAT GAA TG-3' & & \\
\hline \multirow[t]{2}{*}{$D 6 R k f 14$} & L: 5'-ACC TCA AAC TAG ACC CTG AAG-3' & 58 & 231 \\
\hline & R: 5'-TAA AGA AAA TGT ATT GAT CC-3' & & \\
\hline \multirow[t]{2}{*}{$D 6 R k f 15$} & 1: 5'-GCT CAT GCA TGG CAA AGA AG-3' & 55 & 167 \\
\hline & 2: 5'-CAA CTA TTG AGA ATC TCT GTT AG-3' & & \\
\hline \multirow[t]{2}{*}{$D 6 R k f 17$} & B: 5'-TTC ATT GAT GAG TTG AAG CCC-3' & 58 & 284 \\
\hline & C: 5'-AGG AGA TGA GTG AGC AGG ACA-3' & & \\
\hline \multirow[t]{2}{*}{$D 6 R k f 24$} & B: 5'-CTG AGC AGC AAT CAC GTT GT-3' & 58 & 309 \\
\hline & C: 5'-TCA TGC TGA CCC AGA CTC TG-3' & & \\
\hline \multirow[t]{2}{*}{$D 6 R k f 25$} & A: 5'-GAA GGC ACA CAT TCC TAT CTC C-3' & 58 & 250 \\
\hline & B: 5'-GAT AGA CTG GGA GAT GAG TGG-3' & & \\
\hline \multirow[t]{2}{*}{$D 6 R k f 29$} & 3: 5'-TCG GTG TGA TTA GCA TTC ATG-3' & 58 & 157 \\
\hline & 4: $5^{\prime}$-CTG TGG AGG TTG GAC ACT AGC-3' & & \\
\hline \multirow[t]{2}{*}{$D 6 R k f 30$} & 1: 5'-GTT CCC TAA GCC CTA GGT GG-3' & 58 & 178 \\
\hline & 2: 5'-TAG GCC ATA GTC TTT GGG GA-3' & & \\
\hline \multirow[t]{2}{*}{ D6Rkf33 } & 1: 5'-TGC CAG GAA TTT GCA GGT-3' & 55 & 260 \\
\hline & 3: 5'-CAA CAC CAC AAC CAA TGA GC-3' & & \\
\hline \multirow[t]{2}{*}{$D 6 R k f 34$} & 1: 5'-GAC TAA CAG TAA CGT TTA AAG TG-3' & 58 & 220 \\
\hline & 2: 5'-GTA TTA GTA TGT ATT TTT GAC-3' & & \\
\hline \multirow[t]{2}{*}{$D 6 R k f 39$} & 1: 5'-CTA CCA CTT CTG TTG ATC TGT TG-3' & 55 & 310 \\
\hline & 2: 5'-AAG TAA ATA GAG CCT TAG AAA TGG-3' & & \\
\hline
\end{tabular}

facility at the Rockefeller University Laboratory Animal Research Center under standard procedures.

\section{Mouse Genomic DNA Agarose Block and Liquid} DNA Preparation

Mouse spleens were homogenized and embedded in low melting agarose $\left(5 \times 10^{6}\right.$ cells/block). These genomic agarose blocks were then treated with Proteinase $\mathrm{K}$ and $\mathrm{N}$-lauroylsarcosine (Zuo et al. 1992), digested with different restriction enzymes according to the manufacturer's instructions (New England Biolabs), and then analyzed by regular gel electrophoresis or PFGE as described above. Liquid genomic DNA was prepared from homogenized spleens, kidneys, or tails (Lovell-Badge 1987). Genomic DNA samples of EUCIB were obtained from Human Genome Mapping Project (HGMP) Resource Center (UK).

\section{Detection of Polymorphisms}

For simple sequence length polymorphisms (SSLPs), MIT map-pairs were purchased from Research Genetics, Inc. and used for PCR amplification under the recommended conditions. For the novel polymorphism at the D6Rkf3O locus, the same procedures were used by PCR as the MIT map-pairs. Amplified products were separated in a $15 \times 20 \mathrm{~cm} 10 \%$ acrylamide gel (30:1 acrylamide to bisacrylamide) in $0.25 \times \mathrm{TBE}$ at $200 \mathrm{~V}$ for $2.5 \mathrm{hr}$. For restriction fragment length polymorphisms (RFLPs), 5-10 $\mu \mathrm{g}$ of genomic DNA from different parental mouse strains was digested with a variety of enzymes. Southern blots of the digested DNAs were hybridized with labeled probes. Any RFLPs observed were further used to genotype backcross DNA samples. SSCP analysis was used to genotype backcross DNA samples following a protocol described by Vidal-Puig and Moller (1994) using large acrylamide gels with glycerol. The polymorphism for each probe used in this study is listed in Table 1.

\section{Making YAC Sublibraries}

YAC DNA was excised from low melting agarose gel after separation from endogenous yeast chromosomes by PFGE. Purified YAC DNA ( $-500 \mathrm{ng})$ was digested with restriction enzyme EcoRI in the low melting agarose, purified by glass 


\section{A YAC CONTIG OF THE LURCHER LOCUS}

wool centrifugation or $\beta$-agarase I treatment followed by ethanol precipitation, and finally ligated into $1 \mu \mathrm{g}$ of predigested $\lambda$ Zap II/EcoRI/CIAP cloning vector (Stratagene). The ligation mix was packaged (Gigapack Gold; Stratagene) and analyzed under conditions recommended by the manufacturer. A large number of white plaques or recombinant clones were picked into 96-well plates and directly PCR amplified by T3 and T7 vector primers. Each product was analyzed further for development of new STSs and to determine which YACs they map to.

\section{Interspersed Repetitive Sequence PCR (IRS-PCR)}

YACs were amplified using TNK 50 buffer (described above) and $0.5 \mu \mathrm{M}$ of a B1 repeat-specific primer, B1.1 (Simmler et al. 1991). Samples were processed through an initial denaturation $\left(94^{\circ} \mathrm{C}, 4 \mathrm{~min}\right)$, then 35 cycles of denaturation $\left(94^{\circ} \mathrm{C}, 1 \mathrm{~min}\right)$, annealing $\left(45^{\circ} \mathrm{C}, 2 \mathrm{~min}\right)$, and elongation $\left(72^{\circ} \mathrm{C}, 3 \mathrm{~min}\right)$, followed by $10 \mathrm{~min}$ elongation at $72^{\circ} \mathrm{C}$ and storage at $4^{\circ} \mathrm{C}$. PCR reactions were analyzed on $1.6 \%$ regular agarose gels in $1 \times \mathrm{TAE}$ buffer, and individual bands were excised. PCR products were cloned into the pCR II vector (Invitrogen) following isolation from the gel block by passage through glass wool during centrifugation at $12,000 \mathrm{rpm}$. Partial sequencing of the clones yielded one dinucleotide (CA) stretch; marker D6Rkf14 was developed by making primers flanking this repeat (Table 2 ).

\section{Somatic Cell Hybrid Mapping Panel}

$\mathrm{SCH}$ cell lines have been described in detail elsewhere (Bahary et al. 1992). Briefly, macrophages from $\mathrm{A} / \mathrm{He}$ mice or $\mathrm{L}$ cells from a $\mathrm{C} 3 \mathrm{H}$ background were fused with the Chinese hamster cell line E36. Six such hybrid lines, 2A2B1, 2A2C2, 2A2H3, BEM1-4, ECM4C, and R2-24, were obtained and analyzed further for their mouse chromosomal content by karyotypic analysis. Chromosome 6 is present only in lines 2A2B1, 2A2H3, and BEM1-4 but not in lines $2 \mathrm{~A} 2 \mathrm{C} 2, \mathrm{BEM} 1-4$, and $\mathrm{R} 2-24$. These cell lines were kindly provided by Dr. Jeffrey Friedman (Rockefeller University, New York, NY).

\section{ACKNOWLEDGMENTS}

We thank colleagues in our laboratory for their support and comments, particularly Moyosore Onifade and Lindsey Madden for technical assistance, John Gubbay for comments and somatic cell hybrid blots, and Lori Zeltser and William Yang for comments. We particularly acknowledge Drs. Jeffrey Friedman and Andrew Peterson for advice and are grateful to Dr. J. Friedman for somatic hybrid cell lines pICL and pLUS, Dr. M. Rhodes for EUCIB samples, Dr. K. Hunter for filters of YAC libraries, Dr. J.R. Hansbrough for Jrl, and Dr. S.M. Tilghman for YAC library screening. J.Z. is a post-doctoral associate and N.H. is an investigator of the Howard Hughes Medical Institute. P.L.D. is supported by the National Institutes of Health/ National Institute of General Medical Sciences (NIH/ NIGMS) training grant 5T32GMO7739.

The publication costs of this article were defrayed in part by payment of page charges. This article must there- fore be hereby marked "advertisement" in accordance with 18 USC section 1734 solely to indicate this fact.

\section{REFERENCES}

Bahary, N., J.E. Pachter, R. Felman, R.L. Leibel, K. Albright, S. Cram, and J.M. Friedman. 1992. Molecular mapping of mouse Chromosomes 4 and 6: Use of a flow-sorted Robertsonian chromosome. Genomics 13: $761-769$.

Caddy, K.W. and T.J. Biscoe. 1979. Structural and quantitative studies on the normal $\mathrm{C} 3 \mathrm{H}$ and Lurcher mutant mouse. [Review]. Philos. Trans. R. Soc. Lond. B. Biol. Sci. 287: 167-201.

Chen, H., J.C. Pulido, and G.M. Duyk. 1995. MATS: A rapid and efficient method for the development of microsatellite markers from YACs. Genomics 25: 1-8.

Chua, S.C., K. Hennessey, P. Zeitler and R.L. Leibel. 1993. The little (lit) mutation cosegregates with the growth hormone releasing factor receptor on mouse chromosome 6. Mamm. Genome 4: 555-559.

Dietrich, W.F., J.C. Miller, R.G. Steen, M. Merchant, D. Damron, R. Nahf, A. Gross, D.C. Joyce, M. Wessel, R.D. Dredge, et al. 1994. A genetic map of the mouse with 4,006 simple sequence length polymorphisms [see comments]. Nature Genet. 7: 220-225.

The European Backcross Collaborative Group. 1994. Towards high resolution maps of the mouse and human genomes-A facility for ordering markers to $0.1 \mathrm{cM}$ resolution. Hum. Mol. Genet. 3: 621-627.

Gaylinn, B.D., C. von Kap-Herr, W.L. Golden, and M.O. Thorner. 1994. Assignment of the human growth hormone-releasing hormone receptor gene (GHRHR) to 7 p14 by in situ hybridization. Genomics 19: 193-195.

Grobman, A.B. and D.R. Charles. 1947. Mutant white mice. A new dominant autosomal mutant affecting coat color in Mus musculus. J. Hered. 38: 381-384.

Hermanson, G.G., M.F. Hoekstra, D.L. McElligott, and G.A. Evans. 1991. Rescue of end fragments of yeast artificial chromosomes by homologous recombination in yeast. Nucleic Acids Res. 19: 4943-4948.

Lovell-Badge, R.H. 1987. Teratocarcinomas and embryonic stem cells: A practical approach, pp. 153-182. IRL, Oxford, UK.

McBride, O.W., P.A. Hieter, G.F. Hollis, D. Swan, M.C. Otey, and P. Leder. 1982. Chromosomal location of human kappa and lambda immunoglobulin light chain constant region genes. J. Exp. Med. 155: 1480-1490.

Norman, D.J., C. Fletcher, and N. Heintz. 1991. Genetic mapping of the Lurcher locus on mouse Chromosome 6 using an intersubspecific backcross. Genomics 9: 147-153. 


\section{ZUO ET AL.}

Norman, D.J., L. Feng, S.S. Cheng, J. Gubbay, E. Chan, and N. Heintz. 1995. The Lurcher gene induces apoptotic death in cerebellar Purkinje cells. Development 121: $1183-1193$.

Phillips, R.J.S. 1960. "Lurcher," a new gene in linkage group XI of the house mouse. J. Genet. 57: 35-42.

Reeves, R.H., M.R. Crowley, B.F. O'Hara, and J.D. Gearhart. 1990. Sex, strain, and species differences affect recombination across an evolutionarily conserved segment of mouse chromosome 16. Genomics 8: $141-148$.

Silverman, G.A., J.I. Jockel, P.H. Domer, R.M. Mohr, M.P. Taillon, and S.J. Korsmeyer. 1991. Yeast artificial chromosome cloning of a two-megabase-size contig within chromosomal band 18q21 establishes physical linkage between BCL2 and plasminogen activator inhibitor type-2. Genomics 9: 219-228.

Simmler, M.C., R.D. Cox, and P. Avner. 1991. Adaptation of the interspersed repetitive sequence polymerase chain reaction to the isolation of mouse DNA probes from somatic cell hybrids on a hamster background. Genomics 10: 770-778.

Vidal, P.A. and D.E. Moller. 1994. Comparative sensitivity of alternative single-strand conformation polymorphism (SSCP) methods. BioTechniques 17: $490-492$.

Wetts, R. and K. Herrup. 1982a. Cerebellar Purkinje cells are descended from a small number of progenitors committed during early development: Quantitative analysis of lurcher chimeric mice. J. Neurosci.

2: 1494-1498.

1982b. Interaction of granule, Purkinje and inferior olivary neurons in Lurcher chimaeric mice. I. Qualitative studies. J. Embryol. Exp. Morphol. 68: 87-98.

1982c. Interaction of granule, Purkinje and inferior olivary neurons in Lurcher chimeric mice. II. Granule cell death. Brain Res. 250: 358-362.

1983. Direct correlation between Purkinje and granule cell number in the cerebella of Lurcher chimeras and wild-type mice. Brain Res. 312: 41-47.

Zuo, J., C. Robbins, P. Taillon-Miller, D. Cox, and R.M. Myers. 1992. Cloning of the Huntington disease region in yeast artificial chromosomes. Hum. Molec. Genet. 1: $149-159$.

Zuo, J., C. Robbins, S. Baharloo, D. Cox, and R.M. Myers. 1993. Construction of cosmid contigs and high-resolution restriction mapping of the Huntington disease region of human Chromosome 4. Hum. Molec. Genet. 2: 889-899.

Rt:ceived September 7, 1995; accepted in revised form October 26, 1995. 


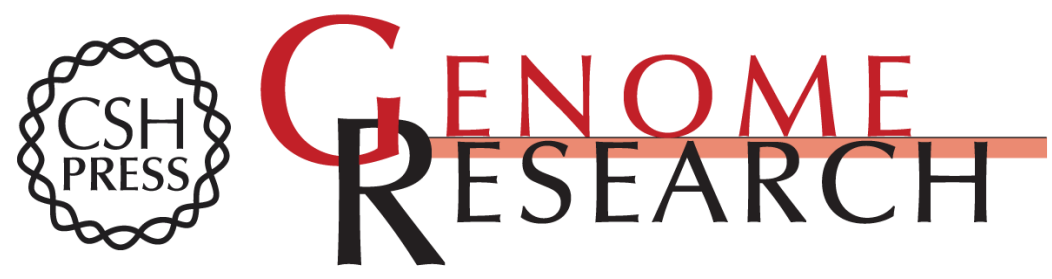

\section{Generation of a high-resolution genetic map and a YAC contig of the Lurcher locus on mouse chromosome 6.}

J Zuo, P L De Jager, D J Norman, et al.

Genome Res. 1995 5: 381-392

Access the most recent version at doi:10.1101/gr.5.4.381

References This article cites 23 articles, 3 of which can be accessed free at:

http://genome.cshlp.org/content/5/4/381.full.html\#ref-list-1

\section{License}

Email Alerting Receive free email alerts when new articles cite this article - sign up in the box at the Service top right corner of the article or click here.

\section{Affordable, Accurate} Sequencing.

To subscribe to Genome Research go to:

https://genome.cshlp.org/subscriptions 